\title{
Redes familiares y el lugar de los varones en el cuidado de la salud materna entre mujeres indígenas mexicanas
}

\author{
Family networks and the role of men in maternal \\ health care among Mexican indigenous women
}

'Doctora en Antropología Social. Investigadora, Consejo Nacional de Ciencia y Tecnología (CONACYT), adscrita al Centro de Investigación y Estudios Superiores en Antropología Social (CIESAS) Pacífico Sur. Oaxaca, México. $\triangle$
RESUMEN Este artículo aborda la mortalidad materna entre mujeres indígenas en México, y las transformaciones en las prácticas de atención durante el embarazo y parto. A través de una investigación cualitativa etnográfica, Ilevada a cabo en el estado de Guerrero, entre 2008 y 2012, cuyo trabajo de campo incluyó entrevistas en profundidad y encuestas a mujeres indígenas, se analiza la medicalización creciente de la reproducción, el papel que juegan las redes familiares en el cuidado durante la gestación, parto y postparto, y la participación de los varones durante el parto, entrando en diálogo con otras investigaciones antropológicas sobre salud materna en México. La antropología médica permite comprender la medicalización creciente de la reproducción en contextos indígenas e identificar la tensión que caracteriza a las redes de cuidado familiar las que, al mismo tiempo que operan como protectoras y movilizadoras de búsqueda de atención, reproducen relaciones de poder marcadas por condiciones de género y generacionales.

PALABRAS CLAVES Antropología Médica; Población Indígena; Mortalidad Materna; Redes Comunitarias; México.

\begin{abstract}
This article reflects on maternal mortality among indigenous women in Mexico and the changes that have occurred in care practices during pregnancy and childbirth. Through ethnographic qualitative research in the state of Guerrero between 2008 and 2012, which included over a year of fieldwork as well as in-depth interviews and surveys with indigenous women, the article analyzes the increasing medicalization of reproduction, the role of family networks in gestation, delivery and postpartum care, and the participation of men during childbirth, in dialogue with other anthropological research on maternal health in Mexico. Medical anthropology allows us to understand the medicalization of reproduction in indigenous contexts and identify the tension that characterizes family care networks, which both operate as protectors and mobilizers in seeking care and reproduce power relations marked by gender and generational conditions.
\end{abstract}

KEY WORDS Medical Anthropology; Indigenous Population; Maternal Mortality; Community Networks; Mexico. 


\section{INTRODUCCIÓN}

La mortalidad materna es un problema de salud pública y un marcador de desigualdad social que impacta en la salud de las mujeres en América Latina y el Caribe. La muerte materna es el fallecimiento durante el embarazo, parto y hasta los 42 días posteriores a la terminación de la gestación, por causas asociadas a esta o agravada por el embarazo y su manejo, que no sea debido a causas accidentales o incidentales ${ }^{(1)}$. La Organización Mundial de la Salud (OMS) reconoce que miles de mujeres en las Américas pierden anualmente la vida ${ }^{(2)}$, principalmente por hemorragias, hipertensión, causas directas y factores preexistentes. Según la Organización Panamericana de la Salud (OPS), una de cada cinco muertes maternas en las Américas obedece a hemorragias durante o posteriores al parto ${ }^{(3)}$, incluso en 2015 se lanzó la iniciativa "cero muertes maternas por hemorragias".

La muerte materna tiene características diferenciadas según el contexto sociocultural, las condiciones económicas y geográficas, de infraestructura de servicios y características de los sistemas de salud, entre otros aspectos. En este sentido, se inserta en el campo de la reproducción, la salud y el género, temas centrales para la antropología médica y el análisis de los procesos de salud-enfermedad-atención-prevención, propios de esta subdisciplina. La reproducción es un proceso sociocultural que no se circunscribe a aspectos fisiológicos o biológicos y está atravesada por múltiples formas de comprender la maternidad-paternidad, la salud y la atención, según órdenes culturales y de género. Asimismo, abarca múltiples ámbitos de la vida cotidiana, incluyendo procesos previos a la gestación, como el cortejo, la selección de pareja, la sexualidad, los rituales de unión y la residencia. También incluye las etapas del proceso reproductivo biológico (embarazo, parto y puerperio), el trabajo productivo posterior al nacimiento, la crianza temprana y las acciones de socialización del nuevo ser en la configuración sociocultural de su grupo ${ }^{(4,5)}$.
Al ser mediada por los órdenes simbólicos y de género propios de cada grupo social, la mortalidad materna presenta manifestaciones diferentes. Así, la cultura y las condiciones materiales y estructurales en las que ocurre construyen una normatividad en torno a la reproducción y a las representaciones y prácticas de prevención, atención o recuperación de la salud durante el embarazo, parto y puerperio. Los grupos sociales definen cuándo deben reproducirse las personas, de qué manera, quiénes participan, deciden e intervienen de manera protagónica o periférica, lo cual sucede a través de transacciones permanentes con actores reguladores de la reproducción, en particular, el Estado, a partir de políticas poblacionales y de salud.

En buena parte de los grupos indígenas y urbanos populares, este proceso reproductivo está marcado por el lugar central que ocupan las redes familiares, especialmente, parejas, suegras, madres, padres y otros integrantes del núcleo familiar, así como la comunidad en general. Con los crecientes procesos de medicalización, el Estado se erige como protagonista en el ámbito de la reproducción biológica y de las prácticas de cuidado desarrolladas durante el embarazo y parto, fortaleciendo la participación de otros actores: personal médico, de enfermería y promoción de la salud, redes sociales y organizaciones, entre otros. En el puerperio este protagonismo estatal desaparece y las prácticas de atención recaen en las redes familiares y comunitarias, pues si bien hay una normatividad que establece el seguimiento a la mujer hasta 42 días posteriores al parto, las acciones de seguimiento y vigilancia desde el personal de salud se centran en el embarazo y parto, posiblemente por considerar que la mayor probabilidad de muerte ya pasó; por insuficiencia de personal o porque las intervenciones en dicho periodo se centran en los recién nacidos y no en la mujer.

Diversas investigaciones en el campo de la antropología médica han indagado las características de la mortalidad materna y los procesos reproductivos en regiones indígenas. En las dos últimas décadas, la literatura etnográfica sobre el tema en México muestra 
la complejidad del fenómeno en estados como Chiapas, Tlaxcala, Oaxaca, Yucatán y en contextos de migración urbana indígena a la Ciudad de México ${ }^{(6,7,8,9,10,11)}$. Varias autoras han evidenciado las transformaciones en la atención del embarazo y el parto, asociadas a la mayor presencia de las instituciones y programas de salud o a los procesos de urbanización y migración. Además, muestran las desigualdades de género como elemento que incide en la mortalidad materna o en la búsqueda de atención, y el papel de las redes sociales, en especial las de parentesco, en las prácticas de autoatención.

\section{ASPECTOS METODOLÓGICOS}

Este artículo se sustenta en mi investigación doctoral Entre la normatividad comunitaria y las instituciones de salud. Procesos reproductivos y salud materna entre mujeres indígenas de la Costa Chica de Guerrero ${ }^{(12)}$ en la cual analicé prácticas de atención durante el embarazo, parto y puerperio, a partir de los límites y posibilidades que establecen las normatividades comunitarias e institucionales en torno a la reproducción.

Asimismo, se indaga el papel de las relaciones sociales -en especial las redes familiares y los hombres como parte de estas- en las prácticas de atención durante el parto, enfocándose en una región pluriétnica de México: la Costa Chica del estado de Guerrero, ubicada al sureste del país; una de las entidades con mayores índices de mortalidad materna durante la última década.

El trabajo de campo fue realizado durante el periodo 2008-2009 y finalizado en 2012, en dos comunidades indígenas de la región de Costa Chica: Nancue Ñomndaa (amuzga) y Na Saavi (mixteca). Además, se diseñó y realizó una encuesta con 100 mujeres entre 15 y 49 años, la cual indagaba aspectos relacionados con la historia sexual y reproductiva de las mujeres, información sobre cada una de sus gestas y partos, mortalidad, morbilidad, tipo de curador al que acudió en cada uno, programas sociales, derechohabiencia, entre otros; además de los datos básicos demográficos. Se seleccionaron 50 mujeres de cada comunidad, con una división por grupo etario que correspondía a la distribución poblacional de la comunidad. Dado que interesaba acceder a la experiencia de atención de mujeres de diferentes grupos etarios, el criterio de selección fue que hubieran experimentado al menos un embarazo a lo largo de su vida, que aceptaran participar y se cumpliera, al final, con el número establecido de encuestadas por cada grupo. Esto permitió construir sus trayectorias reproductivas individuales y por grupo etario. Una ampliación de dicha estrategia metodológica y de los hallazgos por grupo de edad puede consultarse en otro texto de mi autoría donde se profundiza en tales aspectos ${ }^{(13)}$.

Además, se realizaron entrevistas en profundidad con mujeres, hombres, promotoras comunitarias y personal de salud, así como observación participante en los hospitales básicos comunitarios del municipio de San Luis Acatlán -primer nivel de atención- y en el Hospital Regional de Ometepec -segundo nivel de atención-, que brinda servicio a 15 municipios de la región. La investigación incluyó la revisión de programas de política pública, análisis de datos estadísticos oficiales en salud y de recursos asignados, aunque dicha información no se incluye en este texto.

Se solicitó el consentimiento informado verbal a todos los participantes y el protocolo de investigación doctoral fue aprobado por el Comité de Posgrado de Ciencias Antropológicas de la Universidad Autónoma Metropolitana, en 2008, previo a la realización del trabajo de campo. Los resultados fueron presentados en coloquios posteriores del posgrado y aprobados de manera definitiva por el comité dictaminador de la tesis en diciembre de 2013. Los nombres en el texto fueron modificados para garantizar la confidencialidad.

En el abanico de trabajos sobre salud materna inscritos en el marco de la antropología médica crítica, existen trabajos etnográficos; estudios cuantitativos derivados de las bases de datos oficiales en salud; estudios de epidemiología sociocultural; análisis 
de instituciones, políticas públicas o acciones gubernamentales; e investigaciones que abordan las transformaciones de estas prácticas en diversas generaciones y grupos familiares, combinando análisis sincrónicos y diacrónicos.

Este artículo dialoga con algunos estudios claves sobre salud materna en zonas indígenas de México, recuperando hilos conductores de sus hallazgos. El trabajo de Mari Carmen Elu, pionera en la materia, fue realizado en una zona rural de Tlaxcala en los primeros años de la década de 1990, cuando a nivel internacional se impulsaba la Iniciativa por una Maternidad Segura, sentó las bases para un acercamiento cualitativo a la vida de las mujeres fallecidas y a las pautas culturales en las que se insertaban sus historias y prácticas ${ }^{\left({ }^{(9)}\right.}$. La comprensión de las causas socioculturales de la mortalidad materna en áreas rurales, a partir de un acercamiento antropológico, fue fundamental para repensar acciones orientadas a su prevención. Dicho abordaje ha tenido continuidad en antropólogas médicas que documentan las múltiples aristas, tensiones y transformaciones en las representaciones y las prácticas de diversos pueblos indígenas mexicanos durante el embarazo, parto y puerperio.

En el texto inicialmente se analiza la mortalidad materna y la medicalización de la reproducción en regiones indígenas de México; luego se presentan resultados de la investigación en la Costa Chica sobre prácticas de atención en la gestación y el parto para, finalmente, analizar el papel de las redes familiares $y$, en particular, de los varones en dichas prácticas.

\section{MORTALIDAD MATERNA $Y$ MEDICALIZACIÓN DE LA REPRODUCCIÓN}

Según la OMS, la Razón de Mortalidad Materna (RMM) en las Américas, en 2013, fue de 68 fallecidas por cada 100.000 nacidos vivos que, en general, es una razón más baja respecto a la mundial (220 por 100.000 nacidos vivos en el mismo año) y, en el periodo 1990-2013, ocurrió una reducción del $40 \%$ en la mortalidad materna de esta región. Pese a ello, en el año de referencia fallecieron 5.593 mujeres por causas asociadas a la gestación, según el reporte de indicadores básicos de salud en las Américas 2015 $5^{(3)}$. Los datos existentes sobre mortalidad materna en América Latina presentan diferencias entre las fuentes que dificultan establecer con certeza el número de fallecidas. Un informe de 2014, realizado por la OMS, Unicef, UNFPA y Banco Mundial habla de 9.300 mujeres $^{(14)}$. Datos de la OMS ${ }^{(15)}$ señalan que en 2015 hubo 5.593 fallecidas. Sin embargo, este dato aún no incluye la información de varios países que no han reportado o lo han hecho de manera preliminar. La OMS hace estimaciones de la mortalidad materna, aplicando un factor de corrección que permitiría hablar, para 2013, de 7.300 fallecidas para América Latina y el Caribe.

La desigualdad en la distribución de la mortalidad materna es un problema serio; los países con razones de mortalidad materna más bajas en la región son Canadá, con 11 por 100.000 nacidos vivos; Uruguay con 14, Chile con 22 y EE.UU. con 28. Mientras que los países con razones más altas son Haití con 380, Guyana con 250, Bolivia con 200, Guatemala con 140 y Surinam con $130^{(2)}$. Algunos países como Perú, Brasil, El Salvador, Barbados y Ecuador tuvieron descensos significativos en su mortalidad de 1990 a 2013 e ingresaron al grupo de países con baja mortalidad materna; es decir, menos de 100 por 100.000 nacidos vivos. Otros países como México, Belice, Chile y Santa Lucía, ya se encontraban en ese grupo y siguieron reduciendo en más del $40 \%$ la mortalidad materna durante este periodo.

Los datos globales son una radiografía general, pero ocultan las desigualdades regionales y por sectores sociales en cada país. En México, en 2014, la RMM a nivel nacional fue de 38,9 por 100.000 nacidos vivos, pero hay estados que superan ese indicador: Durango con 71,2 , Chiapas con 68,1 , Hidalgo con 65,5 , Guerrero con 58,7 y Chihuahua con $56,5^{(16)}$ y, en varios casos, son entidades 
con elevados niveles de pobreza y precarios indicadores de desarrollo humano. Llama la atención el caso de Durango porque, a pesar de ser un estado del norte, tradicionalmente ha presentado razones de mortalidad materna que superan la nacional y ello está asociado, al igual que Chihuahua, a la alta dispersión geográfica y dificultades de acceso en los municipios de la Sierra. Aunque tiene relativamente pocos decesos, su RMM se incrementa por una menor densidad poblacional. En 2014, Durango tuvo 25 decesos por causas obstétricas y una RMM de 71,2, que resulta la más elevada de su propio registro histórico y es el primer año que su RMM supera a entidades del Sureste como Chiapas, Guerrero, Oaxaca o Veracruz ${ }^{(16)}$.

La literatura sobre el tema revela que, además de la pobreza y las dificultades geográficas de acceso, ciertos grupos poblacionales enfrentan condiciones más adversas, asociadas con procesos de racialización y desigualdades derivadas de su identidad étnica. Las poblaciones indígenas son uno de los grupos más vulnerables y en estas regiones la RMM se eleva de manera considerable. En América Latina, los países con mayor población indígena, como Guatemala, Bolivia, Perú o Ecuador tienen altas razones de mortalidad materna y en zonas indígenas se incrementan más ${ }^{(17)}$.

Graciela Freyermuth ha documentado que en los Altos de Chiapas las mujeres tzotziles de Chenalhó mueren con una frecuencia tres veces mayor que el resto de las mexicanas y dos veces más respecto de otras chiapanecas ${ }^{(6)}$. Esto persistía en 2013, al comparar indígenas con no indígenas ${ }^{(18)}$. Lo mismo ocurre con población afrodescendiente en países como Haití o Brasil, donde la mortalidad materna se concentra en mujeres negras y los procesos de racialización en la atención brindada por el personal de salud se constituyen en formas de violencia institucional, como lo muestra Cecilia MacCallum en su trabajo sobre mujeres adolescentes negras atendidas en la maternidad de un hospital público en Bahía ${ }^{(19)}$.

La asociación entre pobreza y etnicidad en relación con la mortalidad materna se articula con estrategias orientadas a la expansión de la biomedicina y la atención del parto por personal de salud. Así, se establecieron metas internacionales suscritas por los ministerios de salud, dirigidas a alcanzar el $100 \%$ de atención institucional del parto. Las disposiciones hacia la población indígena oscilan entre declaraciones a favor del respeto a las características culturales, a sus cosmovisiones y prácticas de salud, e incluso hacia la implementación de algunas estrategias de adecuación intercultural, mientras que en la práctica la apuesta es por un avance del sistema biomédico. Esta expansión no solo no es rechazada, sino que es demandada por las poblaciones indígenas que piden atención y productos biomédicos. Lo que rechazan, señala Menéndez, es el tipo de relación médico/paciente, el maltrato del personal de salud y el racismo institucional ${ }^{(20)}$.

En las zonas indígenas de México, pese a los recientes recortes presupuestales, hay una presencia cada vez mayor del Estado en el proceso reproductivo y el cuerpo de las mujeres, a través de programas, acciones, recomendaciones y políticas públicas. Al embarazo, parto y puerperio se suman una serie de estrategias y discursos permanentes relacionados con el control de la natalidad, la promoción de familias menos numerosas, la reducción en el número de hijas e hijos y el mayor uso de métodos anticonceptivos de larga duración. Estos discursos que circulan desde las instituciones, los funcionarios gubernamentales, los organismos o las fundaciones internacionales y en organizaciones de la sociedad civil, conforman un conjunto de iniciativas destinadas a modificar comportamientos reproductivos de las mujeres.

Sus efectos se observan en trasformaciones importantes en los saberes de mujeres y hombres de diversos pueblos indígenas y se expresan con mayor contundencia en las generaciones de menor edad. Así, procesos naturales de la vida como el embarazo y el parto, son medicalizados y convertidos en objeto de intervenciones especializadas desde el ámbito de la medicina profesional ${ }^{(21)}$. Se articula la regulación estatal de la 
reproducción, con el saber médico, potenciando así la medicalización.

Actualmente nueve de cada diez partos registrados en México son atendidos en instituciones de salud y más del $94 \%$ de las mujeres acude a consulta prenatal con personal de este ámbito. La institucionalización del parto y la concentración de atención biomédica es evidente pues, en 2015, ocurrieron 2.080.543 nacimientos en el país, de los cuales solo $23.650(1,13 \%)$ fueron atendidos por parteras tradicionales empíricas ${ }^{(22)}$. En ese mismo año, en Guerrero, hubo 61.970 partos y $1.530(2,46 \%)$ ocurrieron con parteras ${ }^{(20)}$. En entidades con alta población indígena, como Chiapas, Veracruz, Puebla, Guerrero y Oaxaca, el porcentaje atendido por parteras se incrementa respecto de la media nacional. Sin embargo, el análisis en Costa Chica con mujeres na saavi (mixtecas) y nancue ñomndaa (amuzgas), confirma la expansión del modelo biomédico, sobre todo, entre mujeres de menor edad que inician su vida reproductiva(5).

En Guerrero, la investigación documentó el predominio del parto hospitalario entre las jóvenes pues ocho de cada diez encuestadas entre 15 y 24 años, tuvieron su último parto en una institución de salud y el $20 \%$ en sus casas. En contraste, entre las mujeres de 25 años en adelante, seis de cada diez optaron por la atención en casa con partera, solas o con sus parejas. Las de mediana edad (25-34 años) y las mayores de 35 años se inclinan por parto en casa y tienen una trayectoria marcada por la terapeútica tradicional o la autoatención. Las jóvenes presentan elevados porcentajes de parto hospitalario, lo que se vincula con el inicio de su vida reproductiva en un contexto de expansión del modelo biomédico y de institucionalización de la atención, que termina naturalizándola. Entre las explicaciones más frecuentes de las jóvenes para esta preferencia, se apelaba a la mayor seguridad que les generaba la atención en el hospital, así como la indicación del personal de salud durante las consultas prenatales para acudir a una institución de salud al momento del parto. Esta representación de mayor seguridad es compartida por varones jóvenes, lo cual potencia la búsqueda de atención biomédica. Finalmente, es necesario señalar un incremento significativo en el número de mujeres cuyo parto se resolvió mediante cesárea y esto es particularmente fuerte entre primigestas y segundigestas. La mayoría de ellas son clasificadas como de riesgo durante el embarazo y por tanto canalizadas a una institución hospitalaria. Este conjunto de fenómenos permite comprender la expansión biomédica entre las mujeres jóvenes.

Esto coincide con investigaciones antropológicas sobre el tema, como el trabajo de Freyermuth en los altos de Chiapas, que evidencia formas de medicalización en este contexto pues, aunque continúa la preferencia de acudir a agentes de salud indígenas especializados, como j'iloles, $j$ 'loktor ja 'jchi'iltic, también se asisten con personal médico, independientemente de que posean una cosmovisión indígena u occidental:

La medicalización del parto, representada sobre todo por la apropiación en el uso de medicamentos de patente como la oxitocina, se da con mayor frecuencia entre las mujeres jóvenes o de la generación de iniciación. Las mujeres que actualmente cursan la vejez o la etapa de predominio manifestaron que no utilizaban ni utilizaron medicamentos ni atención médica o de las parteras para sus partos. ${ }^{(6)}$

Paola Sesia y Matías Sachsse documentaron el desmantelamiento del primer nivel de atención en comunidades rurales de Oaxaca y un elevadísimo número de referencias al segundo nivel de atención, que influye en la sobresaturación de los servicios obstétricos de los hospitales y disminuye la capacidad de atención de emergencias obstétricas, afectando de forma negativa la calidad de la atención ${ }^{(10)}$.

En Yucatán, Judith Ortega muestra la expansión de la biomedicina entre población maya y la reducción de servicios de salud tradicionales, en especial las parteras empíricas $^{(7)}$. La preferencia por recursos biomédicos es clara en las generaciones más jóvenes. 
La autora aborda el papel de los hombres durante el embarazo y parto, evidenciando que, con el incremento de la atención en unidades del Instituto Mexicano del Seguro Social (IMSS) y la influencia de la ideología biomédica, ellos han reducido su participación en las actividades de sobada o durante el parto ${ }^{(7)}$.

Zuanilda Mendoza analiza los cambios en el proceso reproductivo entre dos generaciones de familias triquis migrantes de Oaxaca a la Ciudad de México y describe las prácticas utilizadas por hombres y mujeres durante el embarazo, el parto y el puerpe$\mathrm{rio}^{(23)}$. Muestra diferencias generacionales respecto a la atención del embarazo y el tipo de parto pues en las historias reseñadas, los nueve nacimientos que tuvo la mujer de mayor edad ocurrieron en su casa y acompañada solo de su marido, mientras que la más joven atendió su parto en el hospital ${ }^{(23)}$. Asimismo, hay diferencias en el manejo de la placenta, que siempre fue enterrada por la pareja mayor para evitar enfermedades de los recién nacidos y, en el caso de la otra pareja, desechada por el hospital. En estas historias, el puerperio fue un momento central de cuidado que retorna al ámbito familiar, con el seguimiento de madres, suegras, cuñadas, esposos, familiares y parteras:

El parto es el período donde la práctica médica alópata ha ganado espacios, quizá por ser el periodo más temido donde se reconoce el riesgo de muerte para la madre y el niño. Mujeres y varones de la segunda generación tienen una representación de la capacidad técnica de los profesionales de la salud como el elemento de mayor certidumbre en la resolución del parto, asegurando así la sobrevivencia. No se pone en cuestionamiento una atención institucional, impersonal, fuera de su espacio cotidiano y sin la compañía familiar: en lo que centran su elección es en la capacidad técnica del profesional y en los recursos que puede utilizar para asegurar su eficacia. ${ }^{(23)}$
En Guerrero, opera la misma lógica de asignar mayor confianza a la atención biomédica, en caso de que exista algún problema durante el parto. Sin embargo, persisten las quejas de las mujeres respecto a la atención recibida, la ausencia de personal que hable en su lengua, el no respeto a sus formas tradicionales de atención, sus rituales de protección o formas de parir. En coincidencia con lo señalado por Freyermuth para Chiapas $^{(6)}$, la experiencia de los hombres devino en cambios en las prácticas de acceso a servicios de salud y la medicalización del proceso reproductivo, pues los jóvenes más escolarizados o cercanos al mundo urbano impulsan la atención de sus parejas en unidades de salud.

Durante muchas ocasiones, en el trabajo de campo, acompañé a jóvenes varones que esperaban afuera de los hospitales de la región, a sus esposas o parejas mientras daban a luz adentro, pues no está permitida la presencia de ningún familiar o acompañante una vez que la mujer es ingresada. En todos los casos se trataba de hombres jóvenes de menos de 25 años quienes junto con sus parejas habían optado por atenderse en el hospital. En otros casos, estaban acompañados de sus padres o suegros, quienes compartían esa decisión "para evitar que se complique" o "para no meternos en problemas con los papás de la muchacha si le pasa algo". En una ocasión, mientras acompañaba a una pareja joven que acudió a realizar el trabajo de parto a la Casa de la Mujer Indígena, un lugar atendido por parteras tradicionales, les pregunté dónde querían atenderse y fue él quien respondió:

\section{...vamos a estar aquí con las parteras mientras arrecian los dolores, pero ya al momento del parto nos vamos al hospi- tal porque es más seguro.}

Lo interesante es que posterior al parto muchos de estos varones jóvenes realizaban junto a sus padres o suegros los rituales propios de enterramiento de la placenta que es una actividad mayoritariamente masculina. 


\section{La reproducción de la vida en la Costa Chica de Guerrero}

El censo poblacional más reciente, del año 2010, reportó que la Costa Chica de Guerrero, una zona de 180 kilómetros de costa sobre el Pacífico, con montañas y valles, estaba habitada por casi quinientas mil personas ${ }^{(24)}$. Entre sus colinas, verdes durante el invierno y terracotas en verano, transitan mujeres y hombres curtidos por el sol. Na saavi (mixtecos), me 'phaa (tlapanecos), nancue ñomndaa (amuzgos), afrodescendientes y mestizos, se encuentran en este vasto territorio conformado por 15 municipios Ayutla de los Libres, Azoyú, Copala, Cuajinicuilapa, Cuautepec, Florecio Villareal, Igualapa, Juchitán, Marquelia, Ometepec, San Luis Acatlán, Tecoanapa, Tlacoachistlahuaca, Xochistlahuaca ${ }^{(25)}$.

La población indígena se concentra en los municipios de la montaña, mientras que en la franja cercana a la costa se ubica la población afrodescendiente. Las principales actividades económicas de la región son agrícolas de autoconsumo, comercio, producción pecuaria, pesquera y de artesanías ${ }^{(26)}$. En las comunidades de la montaña, se ve a las mujeres na saavi con sus faldas de colores vivos marcadas al final por acabados de diseños geométricos y huipiles o blusas tradicionales hechas por ellas, con bordados de toda clase de animales, pájaros, guajolotes o ardillas.

De brazos fuertes, tanto hombres como mujeres na saavi y ñomndaa, inician su vida productiva a muy temprana edad en las labores de campo o en oficios domésticos, aunque los ingresos no alcanzan para satisfacer las necesidades básicas. Esto explica, parcialmente, el aumento de la migración en la región, tanto entre na saavi, con una larga historia de migraciones temporales a los campos agrícolas del norte de México o a EE.UU., como de otros pueblos indígenas que se desplazan a lugares más cercanos - ciudades del estado y la capital del paíspara realizar labores de construcción o de empleo doméstico.

Las uniones entre parejas se producen a temprana edad, las normas comunitarias señalan que, entre las mujeres, se espera ocurra entre los 15 y los 16 años. El periodo intergenésico promedio es de dos años, por lo cual hacia los 25 años ya tienen cuatro o cinco hijos y muchas optan, o son presionadas por el sector salud, para usar métodos anticonceptivos permanentes. Para otras mujeres, el periodo de gestaciones se puede extender hasta después de los 30 años, lo cual implica casi tres quinquenios de vida dedicados a las labores reproductivas: embarazo, parto y puerperio, lactancia procesos de crianza, cuidado y socialización de los menores por parte de las mujeres.

De manera creciente, durante el embarazo se han incorporado prácticas biomédicas, como la consulta prenatal, los exámenes de laboratorio y los ultrasonidos gestacionales, que conforman el paquete básico de atención ofrecido a las gestantes, incluso para aquellas sin seguridad social que solo cuentan con el Sistema de Protección Social a la Salud, conocido como Seguro Popular, programa que entró en vigor en el año 2004, al cual se afilia la población abierta atendida por la Secretaría de Salud y sus instancias en cada entidad federativa.

Las mujeres de la región y de otras zonas indígenas conjugan prácticas tradicionales de atención con parteras empíricas -como las revisiones, sobadas y manteadas-, con este paquete de intervenciones básicas, el cual usan por presión de las instituciones a través de los programas de transferencia condicionada y porque consideran que les da mayor seguridad en el seguimiento de su embarazo. Esta relación con nuevas tecnologías diagnósticas ha ganado tanto espacio que muchas parteras piden a las gestantes que se realicen los exámenes y el ultrasonido para aceptar atenderlas, una vez que comprueban que todo va bien. Esto coexiste con la presión institucional a las parteras para evitar que atiendan partos.

Como planteó Mendoza para el caso de la comunidad triqui, durante el parto se percibe la mayor hegemonía del sistema biomédico, pues hay una fuerte institucionalización y control de los procedimientos, espacios, tiempos y personal involucrado, los cuales son definidos solo por la institución ${ }^{(23)}$. La 
eliminación del cobro del parto, desde 2009, sumado a los programas de salud materna y los de transferencia condicionada, contribuyen a este incremento.

La atención en una unidad de salud implica que las mujeres y sus acompañantes salgan de la comunidad $y$, si es un procedimiento complejo, se desplacen a la cabecera municipal o al segundo nivel de atención, lo que duplica el tiempo de traslado. Un aspecto que marca la experiencia de varias mujeres indígenas de la Costa es el temor frente a lo desconocido, a las consecuencias de salir de la comunidad, desplazarse a una ciudad que no dominan e insertarse en las lógicas hospitalarias. Parte de este temor se sustenta en la posibilidad de que algo pueda acontecer durante el parto o la estancia hospitalaria y la mujer se encuentre sola y sin una red de apoyo para velar por ella.

\section{DISCUSIÓN: EL PAPEL DE LA RED FAMILIAR EN EL CUIDADO DE LA SALUD DURANTE EL PARTO}

Eduardo Menéndez enfatiza la importancia de considerar en las descripciones y el análisis la perspectiva relacional y los diferentes actores involucrados en los procesos de salud-enfermedad-atención ${ }^{(27)}$. Además, evidencia que existen diversos tipos de relaciones y rituales contemporáneos en los ámbitos laborales, de salud, religiosos y familiares, lo cual es contrario a las perspectivas que plantean la erosión o desaparición de relaciones sociales, considerando a los individuos como seres aislados ${ }^{(27)}$.

Coincido con este autor, en el sentido de que "todo sujeto se constituye dentro de relaciones sociales, y que su trayectoria se dará a través de relaciones de colaboración, ayuda mutua, competencia o de lucha"(13). La inserción en una serie de relaciones sociales y la existencia de rituales contemporáneos en torno a la reproducción no es exclusiva de sociedades rurales, indígenas o de sectores populares, se expresa de diferentes formas en cada contexto cultural y aparece claramente frente a eventos específicos de atención a la salud, en los que se activan estas redes y relaciones. Ahora bien, recupero su Ilamado a no considerarlas naturalmente positivas $y$, por tanto, a mirar las tensiones que las caracterizan, su papel protector de la salud pero, al mismo tiempo, reproductor de relaciones de poder ${ }^{(13)}$.

Aun cuando la medicalización del embarazo, parto y la anticoncepción están centrados en la mujer, como sujeto a controlar, vigilar o atender, este proceso de individualización, e incluso de aislamiento de ella una vez que ingresa al hospital durante el parto, no elimina el papel central de las redes no profesionales y sus cuidados "profanos" en términos de Haro ${ }^{(26)}$. Para las mujeres, esto podría significar la diferencia entre la vida y la muerte.

En caso de emergencia o complicaciones, la decisión del traslado pocas veces corresponde solo a la mujer, pues participan integrantes de su red cercana, en particular, suegras, suegros, pareja, cuñadas, madres o padres; también puede incluir vecinos, familiares más lejanos, agentes locales de salud o autoridades comunitarias. Estas redes se activan en momentos claves y pueden operar como factor protector de salud pero también ejercen relaciones de poder marcadas por desigualdades de género y generación, que contribuyen a poner en riesgo la vida o conducir a la muerte, debido a la negativa de búsqueda de atención ${ }^{(6,28)}$.

Menéndez es enfático en cuestionar que las relaciones sociales son "buenas en sí", otorgándoles un papel protector o benéfico respecto a los procesos de salud-enfermedad-atención ${ }^{(13)}$. La literatura antropológica sobre salud materna en población indígena de México documenta que muchas situaciones de violencia contra las mujeres, incluyendo las ocurridas al momento del parto, están asociadas a las relaciones de tipo microsocial en el ámbito familiar, marcadas por relaciones de subordinación y poder intra e intergenérico.

Así como madres, suegras y cuñadas pueden promover la búsqueda de atención para las gestantes e involucrar a los varones, 
también se constituyen en sujetos que ejercen poder hacia las más jóvenes y pueden decidir no buscar atención ante una emergencia, en especial si las gestantes son huérfanas o no cuentan con una red de apoyo propia. En ciertos casos, las redes horizontales, como las promotoras comunitarias en salud, tienen un papel fundamental, sobre todo si se trata de una complicación y asumen un lugar protagónico de mediación entre el mundo indígena y el modelo biomédico. Estos hallazgos son posibles mediante análisis que integran el género como variable analítica.

Por lo tanto, se considera que las relaciones no son intrínsecamente positivas o protectoras de la salud y deben ser analizadas contextualmente, pues tienen diferentes matices en función del padecimiento, de las personas involucradas y de las relaciones de poder existentes. En el primer parto de una joven amuzga, que al momento de dar a luz tenía 14 años, sus suegros se negaron a trasladarla para recibir atención durante un trabajo de parto prolongado con apoyo de la partera:

Cuando estaba viviendo con ellos tuve mi primer niño, nunca me apoyaron, lo único que decían que la mujer tiene que sufrir para tener sus hijos. Sí, porque mi suegra ella dijo que no está gorda, está delgada y todos sus hijos de ella nacieron normal, ella tenía que hacer fuerzas y así me tocaba a mí, aunque quedé toda magullada después del parto. Hubiera querido que me sacaran, pero mi esposo hizo lo que decían sus papás. (Lizbeth. ñomndaa, 26 años, cinco hijos)

Al mismo tiempo Ana, na saavi de 15 años, fue reconvenida por su suegra para acudir al hospital a realizarse estudios de laboratorio, pese a que no quería hacerlo. La suegra indicó a su esposo e hijo que buscaran dinero para el traslado de ella y la acompañó todo el tiempo. Por su parte, Lucia, na saavi de 23 años, salvó la vida debido a la movilización de toda su familia de alianza cuando presentó una amenaza de aborto de su segundo hijo.
Trabajos antropológicos recientes, realizados por integrantes del Seminario de Género, Salud y Reproducción que coordinamos junto con la doctora Ángeles Sánchez y el cual incluye estudiantes de maestría y doctorado de la Universidad Autónoma Metropolitana, Universidad Nacional Autónoma de México y Centro de Investigaciones y Estudios Superiores en Antropología Social, muestran experiencias similares en contextos urbanos. Graciela Muñoz aborda la maternidad entre mujeres en situación de calle en la Ciudad de México y evidencia que los lazos sociales con la familia de origen se activan durante la gestación ${ }^{(29)}$. En muchos casos las familias, a menudo expulsoras de estas jóvenes por situaciones de violencia y abuso sexual al interior de la unidad doméstica, asumen la crianza y socialización de las y los hijos, mientras las madres regresan a la calle ${ }^{(29)}$.

La investigación realizada por Irma Romero aborda casos de mujeres que fallecieron por una complicación obstétrica severa en el Instituto Nacional de Perinatología (INPER), unidad especializada de tercer nivel, y retoma lo que ocurre con las y los menores, así como con la familia, después del deceso $^{(30)}$. En todos los casos fue la familia de la fallecida, quien asumió el cuidado de las y los recién nacidos que sobrevivieron. Algunos de los varones -padres de los menoreslo hicieron durante un tiempo, pero después dejaron estas tareas a madres, suegras, hermanas o cuñadas ${ }^{(30)}$.

Esto coincide con lo reportado por Bianca Vargas con mujeres rurales de la cuenca del lago de Pátzcuaro, en Michoacán, quienes fallecieron por mortalidad materna y cuya descendencia quedó a cargo de abuelas o tías $^{(31)}$. En ambos trabajos se observa el papel de estas redes de parentesco en la búsqueda de atención y su participación activa en la definición del prestador de servicios de salud al cual se acude, como lo muestra Claudia Carrera con parejas urbanas de la Ciudad de México, usuarias del parto humanizado en el ámbito público y privado ${ }^{(32)}$. 


\section{El lugar de los hombres en la atención del parto}

Como han señalado Benno de Kjeizer ${ }^{(33)}$, Figueroa y Franzoni ${ }^{(34)}$, entre otros, el proceso de salud-enfermedad-atención de los hombres ha permanecido relativamente invisible en los estudios, políticas y programas en salud desde una perspectiva de género. Ciertamente, como lo propone Kjeizer, el género no es solo un marcador de inequidad sino un eje explicativo para comprender las distintas formas de entender la salud o el padecer; de enfermar, de morir, de atender y mantener la salud propia y la ajena. También permite comprender las normas estructurantes de las relaciones entre lo femenino y lo masculino, así como la diversidad de arreglos sociales que organizan los lugares ocupados por hombres y mujeres al interior de un grupo, la relación con el cuerpo y las prácticas de atención-prevención que cada quien desarroIla, las cuales, aunque tienen una dimensión individual, indudablemente están marcadas por las relaciones de género. La literatura sobre salud reproductiva $-y$ esta investigación no es la excepción- ha enfatizado la mirada en las mujeres en el análisis de sus representaciones, comportamientos, prácticas sexuales y reproductivas, en el acceso a la toma de decisiones, en el conocimiento y uso de tecnologías anticonceptivas, entre otras. Rojas ${ }^{(35)}$ considera que muchas de las investigaciones en torno a la fecundidad y la reproducción han privilegiado a las mujeres por diversas razones teóricas y metodológicas, a partir de supuestos que ya han sido cuestionados en la demografía y las ciencias sociales. Dicha autora hace un interesante recorrido por los estudios clásicos y actuales sobre la participación de los varones en la reproducción y la paternidad, mostrando investigaciones recientes de tipo cualitativo que dan cuenta de las prácticas, significados, y representaciones masculinas contemporáneas sobre la reproducción.

En el caso de la mortalidad materna hay una centralidad de los análisis en las mujeres, posiblemente, porque son las mujeres quienes enfrentan el riesgo de fallecer. Sin embargo, un aporte central de las ciencias sociales $y$, particularmente, de la antropología, es dar cuenta de la diversidad de actores que influyen en ello y del papel central que ocupan tanto los varones como otras mujeres de mayor edad en las definiciones sobre la reproducción así como en la toma de decisiones respecto a la búsqueda de atención. Sin duda, es necesario acercarnos de manera más profunda y reconocer el rol que juegan los hombres en la asignación de recursos de diversos tipos para la recuperación de la salud en casos de emergencias obstétricas, o las acciones de prevención y mantenimiento de la salud que están a su cargo, tanto en el embarazo, como en el parto y el puerperio.

Mi propia investigación colocó el foco en la experiencia de las mujeres y es evidente el peso que tienen en ella, sin embargo, en el trabajo de campo aparecieron informaciones valiosas sobre acciones de prevención y cuidado que eran claramente responsabilidad de los varones; así como asistir de manera periódica con algún tipo de curador, "tener el valor de parir" o conservar el equilibrio fríocaliente y las restricciones alimentarias, eran responsabilidad de las mujeres. En el caso de Costa Chica, en diferentes momentos del proceso de gestación, los hombres asumen funciones de acompañamiento, cuidado o proveeduría, entre ellas, hacer los rituales de protección durante el embarazo para prevenir o contrarrestar posibles acciones de brujería o maldad, acompañar durante el parto (de manera directa o afuera del hospital), enterrar la placenta después del parto o anteriormente entre los mixtecos, preparar el temazcal en el puerperio. Es decir, son funciones que no se limitan al papel de proveedor económico o a considerarlo como un actor que solo restringe derechos y ejerce violencia.

Al conversar con varones de entre 50 y 70 años, fue clara su presencia durante el parto y el puerperio. Para muchos, los partos ocurrieron en condiciones adversas y sin suficientes recursos de atención, pues en ocasiones vivían en ranchos fuera de la comunidad. Esto propició que les correspondiera llamar a la partera en el momento del parto o hacerse cargo, sosteniendo a la mujer para que 
"hiciera fuerzas", calentando el agua para el baño posterior, recibiendo al bebé al caer, verificando la salida de la placenta, cortando el cordón umbilical y enterrando la placenta luego del alumbramiento, según los procedimientos de cada comunidad.

Por su parte, la mujer preparaba los implementos necesarios para calentar el cuerpo (tés, cobijas), daba a luz, se bañaba a sí misma luego del nacimiento -solo de la cintura para abajo a fin de evitar enfriamientos-, limpiaba al bebé y de inmediato comenzaba a amamantarlo. Estas prácticas de autoatención son relatadas por Ernestina, amuzga de 47 años con 14 gestas, quien tuvo el cuidado de su esposo Enrique:

Solos estuvimos en tres partos, solo los dos. Dejamos que el bebé solo cayera, ya después él me suelta, me apoyo en la silla y él mira si el bebé cayó con todo y placenta. Entonces mira y sale un poquito el bebé y checa que ya baje cualquier líquido. Y sí, ya salió la placenta. Así estuvimos nosotros solos, en el parto de varias de mis hijas y de uno que se me murió. Yo sé que ya es la hora porque sentía que mi bebé ya iba embocando. Ese momento en el que el bebé va embocando y ya no puedo caminar ni para adelante ni para atrás, no puedo sentarme ni acostarme. Entonces ya sé y tiendo trapos, pongo un petate y tiendo trapos que ya no sirven, entonces ahí me hinco para que el bebé no se ensucie, entonces entre lo que me hizo el té, porque aquí la creencia de nosotros es que el cuerpo está muy frío y por eso no avanza el proceso del parto. $Y$ en el tiempo que estuvimos con él en el monte, entonces lo que yo hacía era alistar mi chocolate, mis ampolletas y mis sobres de comino. Si veo que no me apura mucho entonces pongo hacer mi té comino, le pongo media tablilla de chocolate, me tomo mi vaso y al momento me apura el dolor. Así que él ya sabía y cuando ya no tenía ni ganas de ir a poner el té, él me decía: ¿dónde guardas el comino? ¿Dónde guardaste el chocolate para hacerte tu té? $Y$ enfriando y enfriando y yo ya me lo tomaba. $Y$ ya, mientras tanto él se iba alistando, iba buscando la tijera y el hilo para amarrarle el ombligo al bebé y el bote del alcohol. (Ernestina, amuzga, 47 años, siete hijos vivos y 14 gestas)

El uso de oxitocina durante el parto, autoadministrada sin prescripción médica, no es una práctica generalizada en la región como se ha documentado en otras zonas del país; sin embargo, su uso por parte de esta mujer muestra una transformación en los saberes sobre el parto y una combinación de prácticas tradicionales con biomédicas. Los riesgos para la salud derivados de una inadecuada administración, son evidentes.

Durante el puerperio, si no se contaba con familiares o vecinos que pudieran ayudar, el hombre garantizaba la alimentación de su esposa, pero esta parte a menudo era asumida por otra mujer. De acuerdo con los relatos, los hombres conseguían en el monte las hojas necesarias para el baño con hierbas, lo cual implica el reconocimiento de las hierbas y de sus propiedades, saberes que habían adquirido con sus esposas, madres, suegras o con las parteras, como señalaron don Beto, don Rogelio y don Enrique, al preguntarles cómo aprendieron. Una vez seleccionadas las hojas, la responsabilidad de hervirlas y ayudarlas con el baño durante varios días, también los involucraba.

Un elemento interesante de diferencia cultural entre hombres na saavi y ñomndaa es la existencia de parteros varones entre los primeros, lo cual no se observa en la región amuzga. En la comunidad de Buenavista y sus anexos existen cerca de cuatro parteros, que regularmente atienden a las embarazadas, igual que las parteras. La mayoría aprendió con sus suegros o suegras e incluso en la experiencia de atención a los partos de su pareja.

Don Enrique, esposo de Ernestina, es mixteco y aprendió con su suegra, a quien ayudó a atender los partos de su esposa. Después del fallecimiento de su primera cónyuge, se casó con Ernestina (ñomndaa) y ha estado presente en seis partos. Sin ser 
partero, ha atendido por necesidad debido a insuficiencia de recursos de atención, 16 partos a lo largo de su vida, todos hijos suyos con dos esposas:

Yo aprendí con mi suegra que era partera. La mamá de mi primera esposa. $Y$ era de las buenas parteras, solo que en aquellos tiempos no había para que trabajaran con doctor, pero ella sí era buena partera. Yo veía y ella me decía cómo hacer, y así le hacía, al aventón, porque como no sé. Entonces tu suegra te iba indicando, agarra el manto, así haga. Y cuando ella se sienta mal haga así. Así porque ya en esa época ella estaba mal. La primera vez que la ayudé, así nomás en el puro aventón, no pensé si lo estaba haciendo bien o si lo estaba haciendo mal. Quién sabe. Pero yo ayudaba. Sí así fue, en todos los partos de mi esposa estuve ayudando. Buscaba entonces la partera y ahí estoy yo con ellos. Namás la veía, recogía el niño. La sostenía en la cintura, de acá, la abrazaba fuerte en la cintura y ella se sostenía de mi cuello. Ya después, cuando nos salimos y nos vinimos para acá [con Ernestina], me tocó solo con la partera. Ya lo arreglaba yo, también tocaban sus cuidados, no fue con médico. Le daba su té de comino, tablilla de chocolate. Ella me decía ya el remedio está listo, sácalo y me lo tomo. (Don Enrique, na saavi, 70 años)

En varios partos, su atención implicaba la amarrada del cordón umbilical. Según don Enrique lo aprendió al tanteo, pero Ernestina lo señala con mucho orgullo como algo que sabe hacer. Refiriéndose al parto de su primera hija en común narra:

Como dos horas nomás duré con dolor y ya nació. Él, mi marido, le trozó el cordón umbilical, todavía mandó llamar a mi mamá, pero cuando mi mamá llegó ya había pasado todo, ya le había trozado el cordón umbilical. Por eso sí es diestro para amarrar ombligos. Porque hay vez que quedan mal amarrados y empiezan a sangrar, mis bebés quedaron bien amarrados, porque él los deja bien apretados. (Ernestina, amuzga, 47 años, siete hijos vivos y 14 gestas)

Así, hay una presencia importante de hombres como ayudantes durante el parto, en especial con mujeres con gran número de gestas, quienes a menudo han estado solas en casa o con su pareja; así como durante el puerperio, en los baños de hierbas y otras actividades para mantener la salud, lo cual coincide con los hallazgos de otras investigaciones. En los Altos de Chiapas y otras regiones del país, sostienen a las mujeres cuando están paradas o les presionan el útero para ayudar al descenso, mientras ellas están hincadas y ellos sentados. En la costa de Guerrero, los varones las sostienen en las piernas, ambos sentados, o de frente, y ellas hincadas, mientras la partera recibe al niño o a la niña.

Por su parte, Ortega describe con detalle las representaciones de los varones mayas sobre cómo ciertos padecimientos de los recién nacidos correspondían al consumo excesivo de alcohol o tabaco por parte de ellos ${ }^{(7)}$. Además, muestra que los hombres se ocupaban del cuidado físico y emocional durante el embarazo, incluyendo las prácticas sexuales en esta etapa.

Algunas actividades se transformaron, como el temazcal durante el puerperio, que se reemplazó por el baño con agua de hierbas, por lo cual la intervención del varón se reduce, pues esta práctica queda a cargo de la suegra o de otras mujeres. Para los más jóvenes, estos cuidados desaparecen para dar lugar a un acompañamiento durante el parto, en casa u hospitalario, y el apoyo en acciones de cuidado a la mujer y el bebé.

En los hospitales de San Luis y Ometepec se observó que cuando el parto ocurre en este ámbito, la participación de los hombres es de acompañamiento a su pareja durante el proceso, comprando medicamentos, autorizando procedimientos, recibiéndolas a la salida para garantizar el traslado, e interactuando con el personal de salud como interlocutor directo. En muchos casos, el personal 
médico del hospital se dirigió a ellos para explicar o pedir autorización para realizar procedimientos obstétricos o de planificación familiar, dar indicaciones respecto a medicamentos, salidas y pagos

Entre el personal de salud de Costa Chica existe la percepción general de poca presencia masculina en el proceso de cuidado en la gestación. Esta es una de sus principales quejas, pues consideran que varios casos de mortalidad materna o complicaciones se originan por el poco interés de los hombres o sus resistencias culturales para permitir la atención. Sin embargo, la investigación muestra que tienen una presencia activa, aunque han cambiado los escenarios y el modo en que se vinculan, debido a una mayor medicalización del proceso.

En diversos momentos fue posible acompañar a hombres jóvenes que esperaban afuera de los hospitales, mientras sus parejas estaban en labor de parto. Asimismo, gestionaban temas de traslado y presionaban al personal para que las atendieran oportunamente. Es posible que su participación no ocurra en los términos esperados por el personal de salud, pero están presentes, incluso antes de la gestación. Lo anterior coincide con hallazgos en Yucatán ${ }^{(7)}$. la zona mazateca en Oaxaca ${ }^{(36)}$, Veracruz $^{(37)}$. Chiapas ${ }^{(6)} \mathrm{o}$ al analizar casos jurídicos por mortalidad materna ${ }^{(11)}$.

El papel de los varones en el proceso reproductivo es un tema que merece ser profundizado en investigaciones futuras, aun cuando trabajos como los reseñados muestran su participación en diversas formas. Entre las personas de mayor edad, se centraba en los procesos de atención al parto y al puerperio. Hoy esa presencia adquiere nuevas formas. Esta red familiar que protege a la mujer y garantiza su integridad, su salud o la búsqueda de atención durante este periodo, sobre todo, en casos de emergencias, convive con una normatividad de género que justifica el lugar subordinado de la mujer y que produce violencia simbólica, emocional y en ocasiones hasta física. No es mi interés desconocer las violencias físicas y simbólicas que ocurren en el ámbito de la reproducción por parte de los varones, pero también es importante mostrar las prácticas protectoras de la salud que desarrollan cotidianamente. Esta es una veta en la que se debe indagar desde una perspectiva antropológica que incorpore explícitamente un análisis de género, dando cuenta de las tensiones y relaciones de poder genéricas y generacionales, con el fin de pensar los procesos de salud-enfermedad-atención.

\section{CONCLUSIONES}

En este texto reflexioné sobre la atención de la mortalidad materna y cómo permite dar cuenta de los cambios en las prácticas de atención y cuidado durante el embarazo, entre las nuevas generaciones de mujeres y hombres na saavi y nancue ñomndaa de la Costa Chica de Guerrero. Ello obedece, en parte, a la expansión de la biomedicina y la incorporación de sus lógicas entre la población, en especial, entre las y los jóvenes.

Al mismo tiempo mostré la importancia de los lazos sociales, en especial, de las redes de parentesco en las prácticas de atención o la búsqueda de ayuda durante el parto, asumiendo que estas relaciones sociales no son intrínsecamente positivas y pueden operar como factores protectores de la salud y como reproductoras de desigualdades de género. En particular, enfaticé la participación de los varones durante el parto y planteé que, pese a la percepción del personal de salud respecto a su escaso involucramiento, están presentes en las experiencias de las mujeres, tanto las de mayor edad, a quienes acompañaban de manera muy activa, y también entre jóvenes, pues aun cuando la institucionalización del parto los deja fuera, siguen asumiendo formas de participación y acompañamiento que deben reconocerse.

Finalmente, evidencié algunos aportes de la antropología médica para comprender los significados sociales asociados a la reproducción, las manifestaciones del proceso reproductivo y la mortalidad materna en contextos indígenas. Desde el trabajo pionero de Mari Carmen Elu, las investigaciones sobre el tema son diversas, con abordajes teóricos 
y metodológicos basados en el trabajo etnográfico, con análisis de los elementos estructurales e institucionales que inciden en la actual configuración de la reproducción. La antropología médica, combinada con la perspectiva de género, constituye un campo de estudio fértil para pensar los procesos de salud-enfermedad-atención en relación con la salud reproductiva y la maternidad, así como para comprender el efecto diferencial de la socialización de género, en la forma en que son estructurados y normados los lugares ocupados por hombres y mujeres, tanto en sus propias estructuras familiares como a nivel comunitario y respecto de las instituciones de salud.

\section{AGRADECIMIENTOS}

Al Consejo Nacional de Ciencia y Tecnología (CONACYT), por su apoyo para la realización de esta investigación a través de sus becas de doctorado 2007-2010. A las mujeres y hombres indígenas de la Costa Chica de Guerrero que generosamente compartieron sus historias.

\section{REFERENCIAS BIBLIOGRÁFICAS}

1. Organización Mundial de la Salud. Clasificación Internacional de las enfermedades y problemas relacionados con la salud. Washington DC: OMS; 1992.

2. Organización Mundial de la Salud; Fondo de las Naciones Unidas para la Infancia; Fondo de Población de las Naciones Unidas; Banco Mundial. Estimaciones de las tendencias de la mortalidad materna 1990-2013. Génova: OMS; 2014.

3. Pan American Health Organization; World Health Organization. Health information and analysis: Health situation in the Américas, basic indicators 2015. Washington DC: OPS, OMS; 2015.

4. Sánchez-Bringas A. Heurísticas del cuerpo: Una mirada desde América Latina. Ciudad de México: Universidad Autónoma Metropolitana, La Cifra Editorial: 2015.

5. Berrío-Palomo LR. Desigualdades en la procreación: Trayectorias reproductivas, atención obstétrica y morbimortalidad materna en México. Ciudad de México: UAM-Xochimilco, Itaca Editores; 2014.
6. Freyermuth G. Las mujeres de humo, Morir en Chenalhó: Género, etnia y generación, factores constitutivos de riesgo durante la maternidad. Ciudad de México: CIESAS, Miguel Ángel Porrúa; 2003.

7. Ortega J. Género, generaciones y transacciones: Reproducción y sexualidad en Mayas de Yucatán. Zamora: El Colegio de Michoacán; 2010.

8. Mendoza Z. De la casa del nene al árbol de las placentas: Procesos reproductivos, saberes y transformación cultural entre los triquis de Copala en la Merced. [Tesis de Doctorado]. Ciudad de México: Centro de Investigación y Estudios Superiores en Antropología Social; 2004.

9. Elu MC. La luz enterrada: estudio antropológico sobre la mortalidad materna en Tlaxcala. Ciudad de México: Fondo de Cultura Económica; 1998.

10. Sesia P, Sachsse M, Pintado A, Lastra Z. Calidad de la atención obstétrica, desde la perspectiva de derechos, equidad e interculturalidad en centros de salud en Oaxaca. Revista CONAMED. 2012;17(supl 1):4-15.

11. Sesia P. La muerte materna: acciones y estrategias hacia una maternidad segura. Ciudad de México: CIESAS, Comité Promotor por una Maternidad sin Riesgos, ACASAC; 2009.

12. Berrío-Palomo LR. Entre la normatividad comunitaria y las instituciones de salud: Procesos reproductivos y salud materna entre mujeres indígenas de la Costa Chica de Guerrero. [Tesis de Doctorado]. Ciudad de México: Universidad Autónoma Metropolitana-Unidad Iztapalapa; 2013. 
13. Berrio LR. Trayectorias reproductivas y prácticas de atención a la salud materna entre mujeres indígenas de la Costa Chica de Guerrero. In: Sánchez Bringas MA. (coord.). Desigualdades en la procreación: La morbilidad asociada al embarazo, parto y puerperio y las trayectorias de atención de las mujeres mexicanas en el Siglo XXI. Ciudad de México: Universidad Autónoma Metropolitana-Xochimilco; 2014.

14. World Health Organization. Trends in maternal mortality 1990-2013: Executive summary [Internet]. 2014 [citado 12 may 2016]. Disponible en: https://goo.gl/EW7pNe.

15. Organización Mundial de la Salud, Organización Panamericana de la Salud. Situación de Salud en las Américas: Indicadores básicos [Internet]. 2015 [citado 12 may 2016]. Disponible en: https://goo.gl/MDJLmm.

16. Observatorio de Mortalidad Materna en México. Numeralia de mortalidad materna 2014. Ciudad de México: OMM; 2014.

17. Cordero-Muñoz LF, Luna A, Vattuone ME. Salud de la mujer indígena: Intervenciones para reducir la muerte materna. Washington DC: Banco Interamericano de Desarrollo; 2010.

18. Freyermuth G. Determinantes sociales en la mortalidad materna en México. Revista CONAMED. 2016;21(1):25-32.

19. MacCallum C. Raza, etnicidad y sexualidades: Ciudadanía y multiculturalismo en América Latina. Bogotá: Universidad Nacional de Colombia; Universidad del Valle; Centro Latinoamericano de sexualidad y derechos humanos; 2008.

20. Menéndez E. Las enfermedades ison sólo padecimientos?: biomedicina, formas de atención "paralelas" y proyectos de poder. Salud Colectiva. 2015;11(3):301-330.

21. Kleinman A. Writing at the Margins. Discourse between Anthropology and Medicine. Berkeley: University of California Press; 1997.

22. Secretaría de Salud; Dirección General de Información en Salud. Sistema de Certificados de Nacimientos (SINAC), cubos dinámicos [Internet]. 2016- [citado 10 ago 2016]. Disponible en: https:// goo.gl/raDjbH.

23. Mendoza Z. Transformaciones en el saber de dos generaciones de triquis migrantes a la Ciudad de México. Salud Colectiva. 2005;1(2):225-236.

24. Censo de Población y Vivienda [Internet]. Ciudad de México: Instituto Nacional de Geo- grafía y Estadística. 2010 [citado 10 ago 2016]. Disponible en: https://goo.gl/vGgAJQ.

25. Gobierno del Estado de Guerrero; Contraloría General del Estado de Guerrero. Programa de Desarrollo de la Región Costa Chica. Guerrero: Contraloría General del Estado de Guerrero; 2012

26. Haro JA. Cuidados profanos: una dimensión ambigua en la atención a la salud. In: Perdiguero M, Comelles JM. (edit.). Medicina y cultura: Estudios entre la antropología y la medicina. Barcelona: Ediciones Bellaterra; 2000. p. 101-161.

27. Menéndez E. Desaparición, resignificación o nuevos desarrollos de los lazos y rituales sociales. Relaciones Estudios de Historia y Sociedad. 2006;XXVII(107):147-177.

28. Argüello-Avendaño $H$. Mortalidad materna a principios del siglo XXI en los Altos de Chiapas, México: violencia estructural y búsqueda de atención [Tesis de Maestría]. Tarragona: Universitat Rovira i Virgili; 2016.

29. Muñoz G. Trayectorias reproductivas y maternidad en jóvenes en situación de calle. Experiencias de desigualdad y violencia de género [Tesis de Maestría]. Ciudad de México: Centro de Investigaciones y Estudios Superiores en Antropología Social; 2012.

30. Romero I. ¿Quién cuida a los hijos? Las redes familiares en el trabajo reproductivo ante la muerte materna [Tesis de Maestría]. Ciudad de México: Universidad Autónoma MetropolitanaUnidad Xochimilco; 2012.

31. Vargas-Escamilla B. Construcción sociocultural de la muerte materna en la región de la Cuenca del lago de Pátzcuaro, Michoacán: experiencia, trayectoria de atención-desatención y estructura familiar. [Tesis de Maestría]. Ciudad de México: Universidad Nacional Autónoma de México; 2016.

32. Carrera C. Nuestros cuerpos, nuestros partos: apropiación subjetiva de derechos en la atención humanizada del parto en el Distrito Federal [Tesis de Maestría]. Ciudad de México: Universidad Autónoma Metropolitana-Unidad Xochimilco; 2015.

33. De Kjeizer B. Hombres, género y políticas de salud en México. In: Figueroa JG. (coord.). Políticas públicas y la experiencia de ser hombre: Paternidad, espacios laborales, salud y educación. Ciudad de México: El Colegio de México; 2014.

34. Figueroa JG, Franzoni J. Paternidad, espacios laborales, salud y educación a la luz de algunas 
políticas públicas. In: Figueroa JG. (coord.). Políticas públicas y la experiencia de ser hombre: Paternidad, espacios laborales, salud y educación. Ciudad de México: El Colegio de México; 2014.

35. Rojas O. Estudios sobre la reproducción masculina. Ciudad de México: El Colegio de México; 2014.

36. Calixto AM. Los talleres de planificación familiar en comunidades indígenas: las metas institucionales y las voces de sus protagonistas. $10^{\circ}$
Congreso de la Asociación Mexicana de Estudios Rurales; Toluca, México; 2015.

37. Cortés B. El riesgo obstétrico y el uso de la cesárea: prácticas y representaciones sociales de un grupo de mujeres del municipio de Zaragoza, Veracruz, que recibieron atención obstétrica en las instituciones públicas de salud. [Tesis de Maestría]. Ciudad de México: Centro de Investigaciones y Estudios Superiores en Antropología Social; 2016.

FORMA DE CITAR

Berrío Palomo LB. Redes familiares y el lugar de los varones en el cuidado de la salud materna entre mujeres indígenas mexicanas. Salud Colectiva. 2017;13(3):471-487. doi: 10.18294/sc.2017.1137.

Recibido: 12 de septiembre de 2016 | Versión final: 16 de febrero de 2017 | Aprobado: 28 de marzo de 2017

Este obra está bajo una licencia de Creative Commons Reconocimiento-NoComercial 4.0 Internacional. Reconocimiento - Permite copiar, distribuir y comunicar públicamente la obra. A cambio, se debe reconocer y citar al autor original. No Comercial — Esta obra no puede ser utilizada con finalidades comerciales, a menos que se obtenga el permiso.

http://dx.doi.org/10.18294/sc.2017.1137 